\title{
ON REGULAR ANTI-CONGRUENCE IN ANTI-ORDERED SEMIGROUPS
}

\author{
Daniel Abraham Romano
}

\begin{abstract}
For an anti-congruence $q$ we say that it is regular anti-congruence on semigroup $(S,=, \neq, \cdot, \alpha)$ ordered under anti-order $\alpha$ if there exists an antiorder $\theta$ on $S / q$ such that the natural epimorphism is a reverse isotone homomorphism of semigroups. Anti-congruence $q$ is regular if there exists a quasi-antiorder $\sigma$ on $S$ under $\alpha$ such that $q=\sigma \cup \sigma^{-1}$. Besides, for regular anti-congruence $q$ on $S$, a construction of the maximal quasi-antiorder relation under $\alpha$ with respect to $q$ is shown.
\end{abstract}

\section{Introduction and preliminaries}

This short investigation in Bishop's Constructive Algebra is a continuation of [9] and [10]. Bishop's Constructive Mathematics is developed on Constructive Logic [11] - logic without the Law of Excluded Middle $P \vee \neg P$. Let us note that in the Constructive Logic the 'Double Negation Law' $P \Leftrightarrow \neg \neg P$ does not hold, but the following implication $P \Rightarrow \neg \neg P$ holds even in the Minimal Logic. We have to note that 'the crazy axiom' $\neg P \Rightarrow(P \Rightarrow Q)$ is included in the Constructive Logic. In the Constructive Logic 'Weak Law of Excluded Middle' $\neg P \vee \neg \neg P$ does not hold, too. It is interesting, that in the Constructive Logic the following deduction principle $A \vee B, A \vdash B$ holds, but this is impossible to prove without 'the crazy axiom'. Bishop's Constructive Mathematics is consistent with the Classical Mathematics.

Relational structure $(S,=, \neq)$, where the relation $\neq$ is a binary relation on $S$, which satisfies the following properties:

$$
\neg(x \neq x), x \neq y \Rightarrow y \neq x, x \neq z \Rightarrow x \neq y \vee y \neq z, x \neq y \wedge y=z \Rightarrow x \neq z
$$

we call set. Following Heyting, the relation $\neq$ is called apartness. A relation $q$ on $S$ is a coequality relation on $S$ if and only if it is consistent, symmetric and cotransitive [6]-[8]: $q \subseteq \neq, q^{-1}=q, q \subseteq q * q$, where $*$ is the filled product between relations [5]. Let $\beta$ be a consistent relation on $S$. We put ${ }^{1} \beta=\beta$ and $\left({ }^{n} \beta\right)=\beta * \cdots * \beta$

2000 Mathematics Subject Classification: Primary 03F65; Secondary 06F05, 20M10.

Key words and phrases: Constructive mathematics, semigroup with apartness, anti-ordered semigroup, anti-congruence, regular anti-congruence, quasi-antiorder.

Supported by the Ministry of sciences and technology of the Republic of Srpska, Bosnia and Herzegovina. 
( $n$ factors, $n \in \mathbf{N}$ ). Then the relation $c(\beta)=\bigcap_{n \in N}\left({ }^{n} \beta\right)$, the cotransitive fulfilment of $\beta$, is the maximal consistent and cotransitive relation on $S$ under $\beta$ [5].

Let $(S,=, \neq, \cdot)$ be a semigroup with an apartness [6]-[8]. As in [8], a coequality relation $q$ on $S$ is anti-congruence if and only if it is compatible with the semigroup operation in the following sense

$$
(\forall x, y, z \in S)(((x z, y z) \in q \Rightarrow(x, y) \in q) \wedge((z x, z y) \in q \Rightarrow(x, y) \in q)) .
$$

A relation $\alpha$ on $S$ is an anti-order [9] on $S$ if and only if $\alpha \subseteq \neq, \alpha \subseteq \alpha * \alpha$, $\neq \subseteq \alpha \cup \alpha^{-1}$ (linearity) and

$$
(\forall x, y, z \in S)(((x z, y z) \in \alpha \Rightarrow(x, y) \in \alpha) \wedge((z x, z y) \in \alpha \Rightarrow(x, y) \in \alpha)) .
$$

A relation $\tau$ on $S$ is a quasi-antiorder [9] on $S$ if $\tau \subseteq \neq, \tau \subseteq \tau * \tau$ and

$$
(\forall x, y, z \in S)(((x z, y z) \in \tau \Rightarrow(x, y) \in \tau) \wedge((z x, z y) \in \tau \Rightarrow(x, y) \in \tau)) .
$$

Let $x$ be an element of $S$ and $A$ a subset of $S$. We denote $x \bowtie A$ if and only if $(\forall a \in A)(x \neq a)$, and $A^{C}=\{x \in S: x \bowtie A\}$. If $\tau$ is a quasi-antiorder on $S$, then the relation $q=\tau \cup \tau^{-1}$ is an anti-congruence on $S$. Firstly, the relation $q^{C}=\{(x, y) \in S \times S:(x, y) \bowtie q\}$ is a congruence on $S$ compatible with $q$, in the following sense

$$
(\forall a, b, c \in S)\left((a, b) \in q^{C} \wedge(b, c) \in q \Rightarrow(a, c) \in q\right) .
$$

We can construct the semigroup $S /\left(q^{C}, q\right)=\left\{a q^{C}: a \in S\right\}$ with

$$
a q^{C}=b q^{C} \Leftrightarrow(a, b) \bowtie q, \quad a q^{C} \neq b q^{C} \Leftrightarrow(a, b) \in q, \quad a q^{C} \cdot b q^{C}=(a b) q^{C} .
$$

We can also construct the semigroup $S / q=\{a q: a \in S\}$ with

$$
a q=b q \Leftrightarrow(a, b) \bowtie q, \quad a q \neq b q \Leftrightarrow(a, b) \in q, \quad a q \cdot b q=(a b) q .
$$

It is easy to check that $S /\left(q^{C}, q\right) \cong S / q$. The mapping $\pi: S \rightarrow S / q$, defined by $\pi(a)=a q$ for any $a \in S$, is a strongly extensional epimorphism. Secondly, note that the relation $\alpha^{C}$ is an order relation on set $(S, \neg \neq, \neq)$. If the relation $\neg \alpha$ is an order relation on set $(S,=, \neq)$, then, as for example in [1] when the apartness is tight, $\neg \neq \subseteq=[7]$, the relation $\alpha$ is called excise relation on $\mathrm{S}$. (The notion of anti-order relation is more general than notion of excise relation.)

For a given anti-ordered semigroup $(S,=, \neq, \cdot, \alpha)$ it is essential to know if there exists an anti-congruence $q$ on $S$ such that $S / q$ be an anti-ordered semigroup. This plays an important role for studying the structure of anti-ordered semigroups. The following question is natural: If $(S,=, \neq, \cdot, \alpha)$ is an anti-ordered semigroup and $q$ an anti-congruence on $S$, is then the set $S / q$ an anti-ordered semigroup? A possible anti-order on $S / q$ could be the relation $\Theta$ on $S / q$ defined by the anti-order $\alpha$ on $S, \Theta=\{(x q, y q) \in S / q \times S / q:(x, y) \in \alpha\}$. But is not an anti-order, in general. The following question arises: Is there any anti-congruence $q$ on $S$ for which $S / q$ is an anti-ordered semigroup? The concept of quasi-antiorder relation was defined in [9]. According to [9], if $(S,=, \neq, \cdot, \alpha)$ is an anti-ordered semigroup and $\sigma$ a quasi-antiorder on $S$, then the relation $q$ on $S$, defined by $q=\sigma \cup \sigma^{-1}$, is an anti-congruence on $S$ and the set $S / q$ is an anti-ordered semigroup under anti-order $\Theta$ defined by $(x q, y q) \in \Theta \Leftrightarrow(x, y) \in \sigma$. So, according to the results in 
[9], each quasi-antiorder $\sigma$ on an ordered semigroup $S$ under anti-order $\alpha$ induces an anti-congruence $q=\sigma \cup \sigma^{-1}$ on $S$ such that $S / q$ is an ordered semigroup under anti-order $\Theta$. In [10] we proved that the converse of this statement also holds. If $(S,=, \neq, \cdot, \alpha)$ is an anti-ordered semigroup and $q$ anti-congruence on $S$ and if there exists an anti-order relation $\Theta_{1}$ on $S / q$ such that $\left(S / q,=_{1}, \neq_{1}, \circ, \Theta_{1}\right)$ is an ordered semigroup under anti-order $\Theta_{1}$, then there exists a quasi-antiorder $\tau$ on $S$ such that $q=\tau \cup \tau^{-1}$ and $\Theta_{1}=\Theta$. So, each anti-congruence $q$ on a semigroup $(S,=, \neq, \cdot, \alpha)$ such that $S / q$ is an anti-ordered semigroup induces a quasi-antiorder on $S$. This was the motivation for introduction of a new notion. For that we need the following notion: Let $f$ be a strongly extensional mapping of anti-ordered sets from $(X,=, \neq, \alpha)$ into $(Y,=, \neq, \beta)$. For $f$ we say that it is reverse isotone if

$$
(\forall a, b \in X)((f(a), f(b)) \in \beta \Rightarrow(a, b) \in \alpha)
$$

holds. An anti-congruence $q$ on $S$ is called regular if there is an anti-order " $\theta_{1}$ " on $S / q$ satisfying the following conditions:

(1) $\left(S / q,=_{1}, \neq_{1}, \theta_{1}\right)$ is an anti-ordered semigroup;

(2) The mapping $\pi: S \ni a \mapsto a q \in S / q$ is an anti-order reverse isotone epimorphism.

We call the anti-order " $\theta_{1}$ " on $S / q$ a regular anti-order with respect to a regular anti-congruence $q$ on $S$ and the anti-order $\alpha$.

It is obvious that the regular anti-order on $S / q$ with respect to a regular anticongruence $q$ and to the anti-order $\alpha$ on $S$ is in general not unique. The following questions now naturally arise: Does there exist the maximal regular anti-order on $S / q$ with respect to a regular anti-congruence $q$ on $S$ ? Are all anti-congruences on anti-ordered semigroups regular? In this note, we give a partial answer on the questions above. In Theorem 1 and Corollary 2 we give necessary and sufficient conditions for anti-congruence on an anti-ordered semigroup to be regular. In Theorem 3 we give a construction of the maximal quasi-antiorder on anti-ordered semigroup $S$ induced by a regular anti-congruence $q$ on $S$.

For the necessary undefined notions, the reader is referred to books [2]-[4], [11] and to papers [5]-[8].

Lemma 0. Let $\tau$ be a quasi-antiorder on set $(S,=, \neq)$. Then $x \tau(\tau x)$ is a strongly extensional subset of $S$, such that $x \bowtie x \tau(x \bowtie \tau x)$, for each $x \in S$. Also, the implication $(x, z) \in \tau \Rightarrow x \tau \cup \tau z=S$ holds for each $x, z$ of $S$.

Proof. From $\tau \subseteq \neq$ it follows $x \bowtie x \tau$. Let $y \in x \tau$ and let $z$ be an arbitrary element of $S$. Then, $(x, y) \in \tau$ and $(x, z) \in \tau \vee(z, y) \in \tau$. So, we have $z \in x \tau \vee y \neq z$. Therefore, $x \tau$ is a strongly extensional subset of $S$ such that $x \bowtie x \tau$.

The proof that $\tau x$ is a strongly extensional subset of $S$ such that $x \bowtie \tau x$ is analogous. Besides, the following implication $(x, z) \in \tau \Rightarrow x \tau \cup \tau z=S$ holds for each $x, z$ of $S$. Indeed, if $(x, z) \in \tau$ and $y$ is an arbitrary element of $S$, then $(\forall y \in S)((x, y) \in \tau \vee(y, z) \in \tau)$. Thus, $S=x \tau \cup \tau z$. 


\section{Regular anti-congruences}

In order to obtain the relationship between regular anti-congruence and quasiantiorder on $S$, the following theorem is essential.

TheOREM 1. Let $(S,=, \neq, \cdot, \alpha)$ be an anti-ordered semigroup, $q$ an anti-congruence on $S$. The following statements are equivalent:

(1) $q$ is regular.

(2) There exists a quasi-antiorder $\sigma$ on $S$, such that $q=\sigma \cup \sigma^{-1}$.

Proof. (2) $\Rightarrow(1)$. By Lemma 1 in [9], since $q=\sigma \cup \sigma^{-1}$, we have that the quotient semigroup $\left(S / q,=_{1}, \neq_{1}, \cdot\right)$ is an anti-ordered semigroup with respect to the anti-order $\theta$ defined as $(q x, q y) \in \theta \Leftrightarrow(x, y) \in \sigma$. If $x, y \in S$ and $(q x, q y) \in \theta$, then $(x, y) \in \sigma \subseteq \alpha$. By definition, $q$ is regular.

$(1) \Rightarrow(2)$. Let $q$ be a regular anti-congruence. Then there exists an anti-order relation $\theta$ on the quotient semigroup $\left(S / q,=_{1}, \neq_{1}, \cdot\right)$ such that $\left(S / q,=_{1}, \neq_{1}, \cdot, \theta\right)$ is an anti-ordered semigroup, and $\pi: S \rightarrow S / q$ is a strongly extensional reverse isotone homomorphism of anti-ordered semigroups. Let $\sigma=\{(x, y) \in S \times S:(q x, q y) \in \theta\}$. By [10], $\sigma$ is a quasi-antiorder on $S$ and it is easy to check that $q=\sigma \cup \sigma^{-1}$.

Corollary 2. Let $(S,=, \neq, \cdot, \alpha)$ be an anti-ordered semigroup, $q$ an anticongruence on $S$. The following statements are equivalent:

(1) $q$ is regular;

(2) There exists an anti-ordered semigroup $(T,=, \neq, \cdot, \theta)$ and a strongly extensional reverse isotone homomorphism $\varphi: S \rightarrow T$ such that $q=\{(a, b) \in$ $S \times S: \varphi(a) \neq \varphi(b)\}$.

Proof. $(1) \Rightarrow(2)$. Let $q$ be regular. Then there exists an anti-order relation $\theta$ on the semigroup $S / q$ such that the natural epimorphism $\pi: S \rightarrow S / q$ is a reverse isotone mapping. Then, by [10], there exists a quasi-antiorder $\sigma$ on $S$ such that $q=\sigma \cup \sigma^{-1}$. So, there exists an anti-ordered semigroup $T=\left(S / q,=_{1}, \neq_{1}, \cdot\right)$ under $\theta$ and a strongly extensional reverse isotone homomorphism $\pi: S \rightarrow T$ such that $\sigma=\{(a, b) \in S \times S:(\pi(a), \pi(b)) \in \theta\}$. Further on, we have $q=\{(a, b) \in S \times S:$ $\pi(a) \neq 1 \pi(b)\}$. In fact,

$$
\begin{gathered}
(a, b) \in q \Leftrightarrow(a, b) \in \sigma \vee(a, b) \in \sigma^{-1} \Leftrightarrow(\pi(a), \pi(b)) \in \theta \vee(\pi(a), \pi(b)) \in \theta^{-1} \\
(\pi(a), \pi(b)) \in \theta \cup \theta^{-1}=\neq_{1} \Leftrightarrow \pi(a) \neq_{1} \pi(b) .
\end{gathered}
$$

$(2) \Rightarrow(1)$. Let $T$ be an anti-ordered semigroup under an anti-order $\theta$ and $\varphi: S \rightarrow T$ a strongly extensional reverse isotone homomorphism such that $q=$ $\{(a, b) \in S \times S: \varphi(a) \neq \varphi(b)\}$. Since $\theta$ is an anti-order relation on $T$, then $\neq=\theta \cup \theta^{-1}$ holds. Thus, by Theorem in [10], the relation $\sigma$ on $S$, defined by $(a, b) \in \sigma$ if and only if $(\varphi(a), \varphi(b)) \in \theta$, is a quasi-antiorder relation on $S$. On the other hand, $q=\sigma \cup \sigma^{-1}$. In fact, if $(a, b)$ is an arbitrary element of $q$, then

$$
\begin{aligned}
\varphi(a) \neq \varphi(b) \Leftrightarrow(\varphi(a), \varphi(b)) \in \theta \vee(\varphi(b), \varphi(a)) \in \theta & \Leftrightarrow(a, b) \in \sigma \vee(b, a) \in \sigma \\
& \Leftrightarrow(a, b) \in \sigma \cup \sigma^{-1}=q
\end{aligned}
$$


Let $(\varphi(a), \varphi(b)) \in \theta$. Then $(a, b) \in \sigma \subseteq \alpha$. By Theorem 1, $q$ is regular anticongruence on $S$.

Recall that, by Lemma 0, any class $a q$ of anti-congruence $q$, generated by the element $a \in S$, is a strongly extensional subset of $S$. Besides, we have the following assertion, which is crucial in characterization of regular anti-congruences on an anti-ordered semigroup $(S,=, \neq, \cdot, \alpha)$ : If $q$ is a regular anti-congruence on an anti-ordered semigroup $S$, then for every $q$-class $a q$ in $S$ we have

$$
((x, y) \bowtie \alpha \wedge(y, z) \bowtie \alpha \wedge x, z \in a q) \Rightarrow y \in a q
$$

for any $x, y, z, a \in S$. If $q$ is a regular anti-congruence on a semigroup $S$, then there exists an anti-order relation $\theta$ on $S / q$ such that the natural epimorphism $\pi: S \rightarrow S / q$ is a strongly extensive reverse isotone homomorphism. Besides, there exists a quasi-antiorder $\sigma$ under $\alpha$, defined by $(x, y) \in \sigma \Leftrightarrow(x q, y q) \in \theta$ such that $\sigma \cup \sigma^{-1}=q$. Let $t$ be an arbitrary element of $a q$. Then $(a, t) \in q=\sigma \cup \sigma^{-1}$. Thus $(a, t) \in \sigma$ or $(t, a) \in \sigma$. Hence, we have

$$
\begin{gathered}
(a, t) \in \sigma \Rightarrow(a, x) \in \sigma \subseteq q \vee(x, y) \in \sigma \subseteq \alpha \vee(y, t) \in \sigma \subseteq q \subseteq \neq \Rightarrow t \neq y ; \\
(t, a) \in \sigma \Rightarrow(t, y) \in \sigma \subseteq \neq \vee(y, z) \in \sigma \subseteq \alpha \vee(z, a) \in \sigma \subseteq q \Rightarrow t \neq y .
\end{gathered}
$$

So, in both cases, we have that $t \in a q \Rightarrow t \neq y$. Therefore, $y \bowtie a q$. We have

$$
((x, y) \bowtie \alpha \wedge(y, z) \bowtie \alpha \wedge y \in a q) \Rightarrow x \in a q \vee z \in a q
$$

for any $x, y, a \in S$. Indeed, if $x, y, z, a \in S$ such that $(x, y) \bowtie \alpha$ and $(y, z) \bowtie \alpha$ and $x \in a q$, then $(a, y) \in q=\sigma \cup \sigma^{-1} \Rightarrow((a, y) \in \sigma \vee(y, a) \in \sigma)$. Thus, we have

$$
\begin{aligned}
& ((a, y) \in \sigma \vee(y, a) \in \sigma) \Rightarrow \\
& ((a, x) \in \sigma \subseteq q \vee(x, y) \in \sigma \subseteq \alpha) \vee((y, z) \in \sigma \subseteq \alpha \vee(z, a) \in \sigma \subseteq q) \Rightarrow x \in a q \vee z \in a q .
\end{aligned}
$$

It is not known whether the condition given above on $q$-classes is sufficient for regularity of an anti-congruence on an anti-ordered semigroup.

Example. We consider the anti-ordered set $S=\{a, b, c, d, e, f\}$ under the relation $\alpha=S \times S \backslash\{(a, a),(a, d),(a, e),(b, b),(b, e),(c, c),(c, b),(c, e),(d, d),(d, e),(e, e)$, $(f, f),(f, a),(f, b),(f, c),(f, d),(f, e)\}$. Define a coequality relation $q$ on $S$ by $q=$ $S \times S \backslash\{(a, a),(b, b),(b, c),(b, d),(c, c),(c, b),(c, d),(d, d),(d, c),(d, b),(e, e),(f, f)\}$. Then

$$
\begin{aligned}
S / q=\{a q & =\{b, c, d, e, f\}, b q=\{a, e, f\}, c q=\{a, e, f\}, \\
d q & =\{a, e, f\}, e q=\{a, b, c, d, f\}, f q=\{a, b, c, d, e\}\} .
\end{aligned}
$$

The following relation is an anti-order relation on $S / q$

$$
\begin{gathered}
\theta_{1}=\wp(S) \times \wp(S) \backslash\{(\{f\},\{f\}),(\{f\},\{a\}),(\{f\},\{b, d, c\}),(\{f\},\{e\}),(\{a\},\{a\}), \\
(\{a\},\{b, d, c\}),(\{a\},\{e\}),(\{b, d, c\},\{b, d, c\}),(\{b, d, c\},\{e\}),(\{e\},\{e\})\} .
\end{gathered}
$$

Then $\left(S / q,=_{1}, \neq_{1}, \theta_{1}\right)$ is an anti-ordered set, $q$ is a regular coequality on $S$. If in $S$ we define the internal operation by the table below, then the set $S$ is an anti-ordered semigroup, $q$ is an anti-congruence on $S$. It is easy to check that $q$ is a regular anticongruence on the semigroup $S$. The proof of these facts is straightforward. 


\begin{tabular}{c|llllll}
$\cdot$ & $a$ & $b$ & $c$ & $d$ & $e$ & $f$ \\
\hline$a$ & $d$ & $d$ & $d$ & $d$ & $d$ & $a$ \\
$b$ & $e$ & $e$ & $e$ & $e$ & $e$ & $b$ \\
$c$ & $d$ & $d$ & $d$ & $d$ & $d$ & $c$ \\
$d$ & $d$ & $d$ & $d$ & $d$ & $d$ & $d$ \\
$e$ & $e$ & $e$ & $e$ & $e$ & $e$ & $e$ \\
$f$ & $d$ & $d$ & $d$ & $d$ & $d$ & $f$
\end{tabular}

REMARK. If $\sigma$ is a quasi-antiorder on $S$, then $q=\sigma \cup \sigma^{-1}$ is the minimal regular anti-congruence on $S$ which contains $\sigma$. In fact, if $\rho$ is a regular anti-congruence on $S$ containing $\sigma$, then $\rho=\rho \cup \rho^{-1} \supseteq \sigma \cup \sigma^{-1}=q$.

Let $q$ be a regular anti-congruence on an anti-ordered semigroup $(S,=, \neq, \cdot, \alpha)$. Then there exists anti-order $\theta$ on $S / q$ such that the natural homomorphism $\pi$ : $S \rightarrow S / q$ is reverse isotone. Hence, by [10], there exists a quasi-antiorder $\sigma$ under $\alpha$ such that $q=\sigma \cup \sigma^{-1}$ and $\theta=\{(a q, b q) \in S / q \times S / q:(a, b) \in \sigma\}$. In the following theorem we show that there exists such maximal quasi-antiorder $\tau$ under $\alpha$ and we give a construction of that relation.

THEOREM 3. Let $q$ be a regular anti-congruence on an anti-ordered semigroup $(S,=, \neq, \cdot, \alpha)$. Then there exists the maximal quasi-antiorder relation $\tau$ under $\alpha$ such that $q=\tau \cup \tau^{-1}$ and $\theta \subseteq\{(a q, b q) \in S / q \times S / q:(a, b) \in \tau\}$. This relation is exactly the following relation $c(q \cap \alpha)=\bigcap_{n \in N}(n(q \cap \alpha))$.

Proof. (1) It is clear that $c(\alpha \cap q) \subseteq \alpha \cap q \subseteq q \subseteq \neq, c(q \cap \alpha) \subseteq \alpha$, and that the relation $c(\alpha \cap q)$ is cotransitive [4]. In fact, for cotransitivness we need to prove that $(a, c) \in c(q \cap \alpha) \Rightarrow(\forall b \in S)((a, b) \in c(q \cap \alpha) \vee(b, c) \in c(q \cap \alpha))$, i.e., to prove that

$$
\begin{aligned}
(a, c) \in c(q \cap \alpha) \Rightarrow & \\
(\forall b \in S) & \left((\forall i \in N)\left((a, b) \in\left(^{i}(q \cap \alpha)\right)\right) \vee(\forall j \in N)\left((b, c) \in\left(^{j}(q \cap \alpha)\right)\right)\right) .
\end{aligned}
$$

First, we have

$$
\begin{aligned}
(a, c) \in c(q \cap \alpha) & \Rightarrow(a, c) \in\left({ }^{2}(q \cap \alpha)\right)=(q \cap \alpha) *(q \cap \alpha) \\
& \Rightarrow(\forall b \in S)((a, b) \in(q \cap \alpha) \vee(b, c) \in(q \cap \alpha)) .
\end{aligned}
$$

Second, suppose that the following implication holds for some $n \geqslant 2$

$$
(a, c) \in c(q \cap \alpha) \Rightarrow(\forall b \in S)\left((a, b) \in\left({ }^{n}(q \cap \alpha)\right) \vee(b, c) \in\left({ }^{n}(q \cap \alpha)\right)\right) .
$$

Thus, since the filled product " $*$ " is associative, we have

$$
\begin{aligned}
(a, c) \in c(q \cap \alpha) & \Rightarrow(a, c) \in\left({ }^{2(n+1)}(q \cap \alpha)\right)=\left({ }^{(n+1)}(q \cap \alpha)\right) *\left({ }^{(n+1)}(q \cap \alpha)\right) \\
& \Leftrightarrow(\forall b \in S)\left((a, b) \in\left({ }^{(n+1)}(q \cap \alpha)\right) \vee(b, c) \in\left({ }^{(n+1)}(q \cap \alpha)\right)\right) .
\end{aligned}
$$

Therefore, for any natural number $n$, by induction, we have

$$
\begin{aligned}
&(a, c) \in c(q \cap \alpha) \Rightarrow \\
&(\forall b \in S)\left((\forall i \leqslant n)\left((a, b) \in\left(^{i}(q \cap \alpha)\right)\right) \vee(\forall j \leqslant n)\left((b, c) \in\left(^{j}(q \cap \alpha)\right)\right)\right) .
\end{aligned}
$$


Hence $(a, c) \in c(q \cap \alpha) \Rightarrow(\forall b \in S)((a, b) \in c(q \cap \alpha) \vee(b, c) \in c(q \cap \alpha))$.

(2) Further on, let $a, b, x$ be arbitrary elements of $S$ such that $(a x, b x) \in c(\alpha \cap q)$. Then $(a x, b x) \in q$ and, by compatibility of $\alpha$ and $q$ in $S$, we have $(a, b) \in q$. Suppose that the implication $(a x, b x) \in c(\alpha \cap q) \Rightarrow(a, b) \in\left({ }^{n}(\alpha \cap q)\right), n \in N$, holds for any $a, b, x \in S$. Then, from $(a x, b x) \in c(\alpha \cap q) \in\left({ }^{n+1}(\alpha \cap q)\right)=\left({ }^{n}(\alpha \cap q)\right) *(\alpha \cap q)$ it follows

$$
\begin{aligned}
(a x, b x) \in c(\alpha \cap q) & \subseteq\left({ }^{n+1}(\alpha \cap q)\right)=\left({ }^{n}(\alpha \cap q)\right) *(\alpha \cap q) \\
& \Rightarrow(\forall y \in S)\left((a x, y x) \in\left({ }^{n}(\alpha \cap q)\right) \vee(y x, b x) \in(\alpha \cap q)\right) \\
& \Rightarrow(\forall y \in S)\left((a, y) \in\left({ }^{n}(\alpha \cap q)\right) \vee(y, b) \in(\alpha \cap q)\right) \\
& \Rightarrow(a, b) \in\left({ }^{n+1}(\alpha \cap q)\right) .
\end{aligned}
$$

So, by induction, we have $(a x, b x) \in c(\alpha \cap q) \Rightarrow(a, b) \in c(\alpha \cap q)$.

The other implication $(x a, x b) \in c(\alpha \cap q) \Rightarrow(a, b) \in c(\alpha \cap q)$ can be proved analogously. Therefore, the relation $c(\alpha \cap q)$ is compatible with the semigroup operation in $S$.

(3) Let $\sigma$ be a quasi-antiorder relation under $\alpha$ such that $q=\sigma \cup \sigma^{-1}$ and $\theta=\{(a q, b q) \in S / q \times S / q:(a, b) \in \sigma\}$. Then $\sigma \subseteq \alpha \cap q$ and $\sigma=c(\sigma) \subseteq c(\alpha \cap q)$ because, by Lemma 0.4.2 in [7], the cotransitive fulfillment satisfies the implication $\sigma \subseteq \alpha \cap q \Rightarrow c(\sigma) \subseteq c(\alpha \cap q)$ and, in addition, since $\sigma$ is a cotransitive relation, $\sigma=c(\sigma)$.

So, the relation $\tau=c(\alpha \cap q)$ is the maximal quasi-antiorder under $\alpha$ such that $q=\tau \cup \tau^{-1}$. Indeed, $q=\sigma \cup \sigma^{-1} \subseteq \tau \cup \tau^{-1} \subseteq q$. Besides, it is clear that the relation $\Theta=\{(a q, b q) \in S / q \times S / q:(a, b) \in \tau\}$ is an anti-order relation on $S / q$ such that $\theta \subseteq \Theta$.

COROLlARY 4. Let $q$ be a regular anti-congruence on an anti-ordered semigroup $(S,=, \neq, \cdot, \alpha)$. Then there exists the maximal anti-order relation on $S / q$. This relation is exactly the following relation $\{(a q, b q) \in S / q \times S / q:(a, b) \in c(q \cap \alpha)\}$.

Proof. Let $\theta_{1}$ be a regular anti-congruence on $S / q$ with respect to $\alpha$. Then there exists a quasi-antiorder $\sigma$ on $S$ such that $q=\sigma \cup \sigma^{-1}$ and $\theta_{1}=\{(a q, b q) \in$ $S / q \times S / q:(a, b) \in \sigma\}$. Since $c(\alpha \cap q)$ is the maximal quasi-antiorder with respect to $\alpha$, then $\sigma \subseteq \tau$ holds. Thus, we have $\theta_{1} \subseteq\{(a q, b q) \in S / q \times S / q:(a, b) \in c(\alpha \cap q)\}$. So, there exists the maximal regular anti-congruence with respect to $\alpha$.

\section{References}

[1] M. A. Baroni, Constructive order completeness, UCDMS 2004/12 (www.math.canterbury. ac.nz/php/research/reports/)

[2] E. Bishop, Foundations of Constructive Analysis, McGraw-Hill, New York 1967.

[3] D. S. Bridges and F. Richman, Varieties of Constructive Mathematics, London Mathematical Society Lecture Notes 97, Cambridge University Press, Cambridge, 1987

[4] R. Mines, F. Richman and W. Ruitenburg, A Course of Constructive Algebra, SpringerVerlag, New York, 1988

[5] D. A. Romano, On Construction of Maximal Coequality Relation and its Applications; in: R. Tošić and Z. Budimac (eds.), Proc. 8th Internat. Conf. Logic and Computers Sciences "LIRA '97", Novi Sad, Institute of Mathematics, Novi Sad 1997, 225-230 
[6] D. A. Romano, A Left Compatible Coequality Relation on Semigroup with Apartness, Novi Sad J. Math 29(2) (1999), 221-234

[7] D. A. Romano, Some relations and subsets of semigroup with apartness generated by the principal consistent subset, Univ. Beograd, Publ. Elektroteh. Fak. Ser. Mat. 13(2002), 7-25

[8] D. A. Romano, A note on a family of quasi-antiorder on semigroup, Kragujevac J. Math. 27 (2005), 11-18

[9] D. A. Romano, A note on quasi-antiorder in semigroup, Novi Sad J. Math. 37(1) (2007), 3-8

[10] D. A. Romano, Inducing a quasi-antiorder by anticongruence on semigroup, Novi Sad J. Math., (To appear)

[11] A.S. Troelstra and D. van Dalen, Constructivism in Mathematics, An Introduction, NorthHolland, Amsterdam, 1988

Prirodno-matematički fakultet

78000 Banja Luka

Srpska

Bosnia and Herzegovina

bato49@hotmail.com 